\title{
Agenciamientos socio-jurídicos en mujeres con jefatura de hogar, en contextos de pobreza y vulnerabilidad social ${ }^{1}$
}

\author{
Socio-legal agencies in household female heads living in contexts of poverty and social \\ vulnerability
}

Recibido: 16 de mayo de 2017 - Revisado: 13 de septiembre de 2017 - Aceptado: 23 de noviembre de 2017

\author{
Claudia María García Muñoz ${ }^{2}$ \\ Claudia Alexandra Munévar Quintero ${ }^{3}$ \\ Nathalia Hernández Gómez ${ }^{4}$
}

\section{Resumen}

Este artículo presenta los resultados de la investigación "Agenciamientos y estrategias de empoderamiento socio-jurídico en mujeres con jefatura de hogar, en situación de pobreza y vulnerabilidad social", cuyo objetivo consistió en develar los sentidos que emergen en un grupo de mujeres con jefatura de hogar, en el municipio de Manizales, expuestas a contextos de pobreza y vulnerabilidad social, a partir de la capacidad de agencia que despliegan frente a las condiciones sociales y jurídicas que las rodean. El enfoque de tipo hermenéutico, se llevó a cabo mediante la aplicación de técnicas como la observación participante, entrevistas semi-estructuradas y talleres narrativos con las mujeres participantes. Para la interpretación de dicha información se aplicó el análisis cualitativo de contenido ACC, el cual permitió develar los sentidos dados por dichas mujeres, respecto a sus condiciones de pobreza y vulnerabilidad social y su capacidad de agencia para transformar dichas condiciones. Los hallazgos relacionados con sus agenciamientos socio-jurídicos, se agrupan en cuatro tipos: la cultura del cuidado, la convivencia, desempoderamiento jurídico y tecnología de gobierno. Todos se relacionan con configuraciones sociales determinadas por contextos de pobreza y por relaciones de gubernamentalidad que restringen el desarrollo humano y bienestar de estas mujeres.

\section{Palabras clave}

Agenciamientos socio-jurídicos, cultura del cuidado, gubernamentalidad, desempoderamiento, resistencia.

\begin{abstract}
This article presents the results of the research work titled "Agencies and strategies for socio-legal empowerment in household female heads, living in contexts of poverty and social vulnerability" in the Municipality of Manizales-Colombia. The objective was to reveal the senses that emerge in a group of household women heads who are exposed to contexts of poverty and social vulnerability. The data collected was based on the capacity of agency that they deploy facing the social and legal conditions that surround them. The hermeneutic approach was carried out through techniques and instruments such as participant observation, semi-structured interviews and narrative workshops with the participating women. For the interpretation of this information, the ACC Content Qualitative Analysis was applied, which allowed to understand the senses given by these women regarding the conditions of poverty and social vulnerability, as well as their agency capacity to transform them. The specific findings were grouped into four types: agencies associated with the culture of care; agencies associated with coexistence; agencies as legal disempowerment, and those associated with government technologies. The final results are related to social configurations which are determined by contexts of poverty and by governmental relations that restrict both the human development and well-being of these women.
\end{abstract}

Keywords

Socio-legal agencies, culture of care, governmentality, disempowerment, resistance.
${ }^{1}$ Artículo derivado del proyecto de investigación: "Agenciamientos y estrategias de empoderamiento socio-jurídico en mujeres con jefatura de hogar, en situación de pobreza y vulnerabilidad social, en la ciudad de Manizales". Financiado por la Universidad de Manizales.

${ }^{2}$ Psicóloga, Magister en Educación y Desarrollo Humano, Doctora en Ciencias Sociales Niñez y Juventud. Docente del Centro de Estudios Avanzados en Niñez y Juventud, Manizales, Caldas, Colombia.

Correo electrónico:

cgarcia@umanizales.edu.co (10 https://orcid.org/0000-0001-92477866

${ }^{3}$ Abogada. Especialista en Derecho Administrativo. Magíster en Desarrollo Sostenible y Medio Ambiente. Doctora en Desarrollo Sostenible. Docente de la Universidad de Manizales, Colombia

Correo electrónico:

cmunevar@umanizales.edu.co

(10. https://orcid.org/0000-0002-91906232

${ }^{4}$ Abogada, Especialista en Derecho Administrativo. Magíster en Derecho Público. Docente de la Universidad de Manizales, Colombia.

Correo electrónico:

nhernandez@umanizales.edu.co

(10) https://orcid.org/0000-0003-3029. 8138

Para citar este artículo use: García, C., Munévar, C., y Hernández, N. (2018). Agenciamientos socio-jurídicos en mujeres con jefatura de hogar, en contextos de pobreza y vulnerabilidad social. Civilizar, 18(35), 73-90. doi:10.22518/usergioa/jour/ $\mathrm{ccsh} / 2018.2 / \mathrm{a} 06$ 


\section{Introducción}

La presente investigación buscó develar los sentidos que emergen en un grupo de mujeres con jefatura de hogar, expuestas a contextos de pobreza y vulnerabilidad social, a partir de la capacidad de agencia que despliegan frente a las condiciones sociales y jurídicas que las rodean. A partir de dicha realidad, se identificaron sus agenciamientos para afrontar dicho contexto, explorando tres ejes, los cuales se relacionan con las condiciones que prevalecen en su entorno y que los promueven o restringen, lo cual crea escenarios de potenciación o debilitamiento de la capacidad de agencia. Para abordar dichos ejes, la investigación se concibió bajo una perspectiva epistemológica comprensiva, con un enfoque hermenéutico, desde la cual se preguntó por aquellas pretensiones emergentes que dichas mujeres despliegan como estrategias de afrontamiento, mediante las cuales buscan mejorar sus condiciones de vida y ampliar las oportunidades de decidir y actuar en pro de las mejores formas de vida que pueden darse.

El interés investigativo radicó en la interpretación de los sentidos que estas mujeres construyen respecto a sus condiciones de vida y los agenciamientos socio-jurídicos que despliegan, para buscar la transformación de dichas condiciones de pobreza y vulnerabilidad social, reconfigurando sus horizontes de futuro, a pesar de las fuertes limitaciones de sus oportunidades.

La investigación utilizó técnicas cualitativas como la entrevista semi-estructurada y los talleres narrativos para el trabajo de campo, el cual se desarrolló con tres grupos de mujeres con jefatura de hogar, vinculadas a algunos procesos comunitarios. La información obtenida fue interpretada mediante el análisis cualitativo de datos ACC, el cual permitió reconstruir las narraciones comunitarias, en las que se identificaron los aspectos relevantes relacionados con los tres ejes problemáticos. Los hallazgos y conclusiones que se presentan a continuación hacen referencia específicamente a la interpre- tación de los agenciamientos socio-jurídicos que estas mujeres ponen en marcha, las condiciones que los promueven y las dinámicas en las cuales se despliegan, develando los sentidos imbricados en las visiones y relaciones que han construido con el orden jurídico, a través de la utilización y/o impugnación de los dispositivos legales para la defensa de sus derechos.

\section{Contexto de pobreza $y$ vulnerabilidad social de las mujeres con jefatura de hogar}

Para abordar el papel que juega el contexto de vulnerabilidad social y pobreza, sobre las personas y particularmente, sobre las mujeres de nuestro estudio, es necesario hacer algunas precisiones conceptuales generales. En primer lugar, el contexto representa una noción de inteligibilidad para comprender la compleja relación entre la unidad del todo y la autonomía de sus componentes (Bianciardi, 2009), relación donde queda en cuestión la centralidad de alguna de sus partes, en tanto, ninguna de ellas se constituye de manera independiente a las demás, ni posee una esencia propia que la sitúe por fuera de lo que está presente y revestido por el contexto. En este orden de ideas, el contexto que literalmente significa "tejido con", alude a aquello que está presente, circunda, influencia y envuelve un organismo, un evento, un sujeto, pero estos últimos, no pueden verse separados, como entidades independientes con su propia esencia. Se trata de una definición que implica el proceso mismo de envolvimiento, en el que las partes son coproducidas de manera interdependiente, pero donde cada una de ellas logra su propia autonomía, dentro de este mismo proceso. En suma, el contexto es entendido como "el entrelazamiento de todas las relaciones concretas y contingentes dentro de un todo - entrelazamiento que se genera, por ende, en la historia de las interacciones entre las partes caracterizadas por una autonomía propia” (p. 5).

Los contextos sociales representan no solo la caracterización de unos espacios físicos definidos o de unas tipologías territoriales 
que componen determinado entorno, sino que además develan un proceder histórico y cultural envuelto en una trama económica que dependiendo de sus formas desarrolla condiciones de pobreza y vulnerabilidad, o de desarrollo y prosperidad, representando las formas de vida de los individuos que los habitan. Ahora bien, en el marco de esta investigación, las condiciones de escasez de recursos en contextos urbanos son la expresión de otra forma de la división social, la que separa a los ricos y los pobres, como consecuencia del surgimiento de la propiedad privada, que es una innovación de la vida urbana.

[...] La ciudad misma, desde sus inicios, surgió como resultado de la explotación del trabajo humano, las labores forzadas que se requerían para construir la misma ciudad, no sólo provenían de la esclavización, sino también del monopolio ejercido sobre los recursos básicos para la vida humana (Lezama, 2014, p. 73).

En consecuencia, los contextos de vulnerabilidad social y pobreza, determinan las condiciones de vida de una gran proporción de la población colombiana y latinoamericana y en especial, afectando a muchas mujeres con jefatura de hogar, restringiendo sus oportunidades de desarrollo humano, generando fuertes asimetrías y desigualdad social. Así lo afirman Ramírez y Ziccardi (2008):

La pobreza urbana, la desigualdad y la exclusión social constituyen procesos claramente observables en la mayoría de las ciudades latinoamericanas del siglo XXI. [...] Se advierten nuevas y diferentes formas de exclusión social, las cuales se observan tanto en las prácticas discriminatorias que prevalecen en el mercado de trabajo como en el acceso a la calidad diferenciadas de los bienes y servicios de la ciudad según el origen socio-económico y étnico de la ciudadanía y su lugar de residencia (p. 23).

Lo anterior, tiene implicaciones directas en la comprensión de las configuraciones socia- les, y en este caso particular, las del grupo de mujeres de este estudio, al ser parte de la trama de componentes del contexto socio-jurídico en el que ellas no solo se desenvuelven sino que también son constituidas, en medio de limitaciones para su bienestar. Ahora bien, el contexto de pobreza y vulnerabilidad social, que caracteriza el entorno en el que viven las mujeres con jefatura de hogar de este estudio, se asocia a condiciones de inseguridad y riesgo, convivencia y problemáticas de la infancia y la juventud, las cuales inciden en sus prácticas de vida, sus oportunidades y en definitiva, su bienestar (Elías, 1989).

Este contexto de pobreza y desigualdad, afecta a las personas generando en ellas una mayor vulnerabilidad. Esta puede analizarse desde diferentes sentidos: el primero desde la perspectiva material o física, es decir, aquella atribuible a las condiciones de existencia humana, en segundo lugar, se hace referencia a la social la cual alude al ámbito,

[...] de las desigualdades, y se refiere a la vulnerabilidad por la situación socioeconómica de las personas. [...] Comprende a la vulnerabilidad como la situación de riesgo derivada de las condiciones sociales y económicas, específicamente de las personas que viven con menos satisfactores (Uribe y González, 2007, p. 207).

Ahora bien, la pobreza no es solo un asunto de bajos ingresos, sino de limitación de oportunidades. Por ello es necesario entenderla como "una situación de privación de capacidades y no simplemente de ingresos o necesidades básicas insatisfechas" (Montaño, 2003, p. 365).

Esta restricción de capacidades afecta la garantía de derechos humanos pero además, trasciende aspectos inmateriales relacionados con el ámbito identitario, afectivo y de empoderamiento de los sujetos, en especial de las mujeres con jefatura de hogar. Se trata de la interrelación de diferentes problemáticas sociales que generan contextos de riesgo. Así 
lo expresa Ballén (2007) "hay cuatro fenómenos sociales que van enlazados entre sí: pobreza, hambre, enfermedades y desempleo; y que casi siempre el primero genera los otros tres" (p. 482). Castro afirma que "existen factores asociados a la violencia que la dinamizan, como las rupturas familiares, el mal uso del tiempo, la desintegración de los valores tradicionales, la marginalidad social" (2005, p. 59).

En este sentido, los entornos de estas mujeres empiezan a conformarse como espacios sociales entrópicos, que reflejan diversas problemáticas y que refuerzan las condiciones de vulnerabilidad, restringiendo los agenciamientos que pueden desplegar y con ello, limitando la posibilidad de trasformar sus condiciones de vida conforme a sus propias elecciones y libertades.

El siguiente fragmento de una de las mujeres entrevistadas, lo ilustra:

Los principales problemas, yo veo que es la falta de unión familiar, y la misma drogadicción. Yo creo que más que la falta de dinero, de plata, es la unión familiar, el hablar entre familia, porque muchas veces, yo como madre separada me voy a trabajar, y yo tengo la gran fortuna de que ni hija se queda con mi mamá y mi papá, es criada por alguien, por personas responsables, porque a nosotros nos criaron personas responsables. Muchos no tienen esa oportunidad muchas (mamás) dejan los hijos con la vecina, porque no tiene apoyo de la familia (Comunicación personal, MJH5, E-F4').

En consecuencia, los contextos de las mujeres de este estudio, empiezan a constituir entornos sociales que refuerzan sus condiciones de vulnerabilidad, limitando los agenciamientos que pueden desplegar y con ello, reduciendo la posibilidad de trasformar sus condiciones de vida conforme a sus propias elecciones y libertades.

\section{El agenciamiento como acción de transformación sobre el mundo}

Agenciamiento es un término de uso frecuente en la literatura sociológica y específicamente, en los estudios sobre desarrollo. Sin embargo, se trata de una categoría problemática, en tanto es abordada desde diferentes perspectivas, no desprovistas de una intencionalidad política. Para este estudio, está referido a aspectos que tienen que ver con la competencia de las personas para actuar de manera autónoma e intencionada sobre el mundo. En consecuencia, se trata de un proceso relacionado con la libertad de hacer y transformar las condiciones externas, en función de alcanzar las metas que se tiene razones para valorar (Sen, 1997). Se trata de aquella capacidad mediante la cual las personas pueden hacer frente "exitosamente a las distintas formas de desigualdad, exclusión social y pobreza, mediante la participación efectiva en las elecciones y decisiones políticas que gobiernan su propia vida [...] y en los vínculos sociales requeridos para actuar sobre su entorno y transformarlo" (Posas, s.f.).

Dentro de esta definición se pueden identificar al menos tres perspectivas; la primera de ellas, tiene que ver con el agenciamiento como proceso de subjetivación; la segunda, como proceso de desarrollo, y la tercera, como ontología de la acción social.

Enfocando el agenciamiento como proceso de subjetivación, se señalan la relación cuerpo-mente y la forma como dicha relación "da forma a la experiencia, modela la personalidad y la conexión social, y apoya la agencia en las instituciones políticas, morales, religiosas y terapéuticas" (Mora, 2008). Desde esta óptica, este proceso se obtiene de la mediación entre la capacidad de agencia del sujeto y su contribución a la producción social y política y está constituido por "expresiones de la voluntad e intencionalidad de un sentido particular de existencia individual y colectiva" (Martínez y Cubides, 2012, p. 76). Es decir, se trata en el sujeto, 
de un proceso constituyente que se opone a las condiciones que lo determinan, lo que implica reconocer la capacidad de acción reflexiva e intencionada del sujeto. De hecho, la capacidad de agencia se asocia a la autonomía del sujeto tal como lo propone Kagitcibasi (2005), se trataría de un yo autónomo relacionado, cuyas características se asocian al poder y control que conlleva; a la visión ética personal y colectiva que le subyace y a la responsabilidad que implica para el sujeto. Puede afirmarse que la agencia es la capacidad de los sujetos de crear sus formas de vida, mediante la acción generativa de otras realidades, ejerciendo control y transformando las condiciones objetivas de sus contextos. En este orden de ideas,

El agenciamiento pone en juego, en nosotros y fuera de nosotros, multiplicidades, territorios, fuerzas de composición. Por lo tanto, no se trata de dos identidades en relación [...] sino de agenciamientos múltiples. El agenciamiento no se afirma en la concreción de un ideal, sino en la expresión de una potencia de actuar (Sztulwark y Duschatzky, 2005, p. 205).

El agenciamiento es toda acción que promueve la capacidad política del sujeto, en tanto se trata de acciones potencialmente transformadoras del orden en el que se inscriben. Sin embargo, dentro de un sistema liberal, el agenciamiento está imbricado en tramas del poder en las cuales es producido y desplegado en dos sentidos: como fuerza intencional, o de oposición (de resistencia al poder) (Ortner; citado en Mora, 2008). Como intencionalidad, la agencia es una acción racional, orientada hacia un objetivo dentro de un orden que no se cuestiona; en cambio, como resistencia, es una acción contrahegemónica, de oposición y emancipatoria frente a un orden de dominación; en este caso, se trataría de una acción que busca generar otros posibles alternos al orden establecido. En cualquier caso, la agencia estaría moldeando las subjetividades, en tanto determina las formas como están siendo los sujetos en el mundo.
Ahora bien, el agenciamiento como proceso de desarrollo, es definido desde la teoría de las libertades de Sen (2000), como aquella capacidad efectiva para alcanzar las metas que se tiene razones para valorar. Se trata de aquella capacidad mediante la cual las personas:

[...] pueden enfrentarse exitosamente a las distintas formas de desigualdad, exclusión social y pobreza, mediante la participación en las decisiones que afecten sus vidas, en las decisiones que tienen que ver con las necesidades y expectativas que forjan y en los circuitos relaciones y apoyos para incidir en sus realidades y transformarlas, con un espíritu de tolerancia, respeto y solución negociada de las diferencias (Posas, s.f.).

Por tanto, el agenciamiento hace referencia a un proceso relacionado con la capacidad de elegir y lograr las propias metas y valores y en este sentido, reconoce la correspondencia con la libertad que tienen las personas de hacer $\mathrm{y}$ transformar sus condiciones en función de sus objetivos de desarrollo y sus visiones éticas sobre su bienestar.

Desde un plano estructural, la agencia implica un "movimiento recursivo por el cual las estructuras reproducen al agente y el agente, a su vez, transforma las estructuras. En la agencia en lo que se insiste es pues en la recursividad entre la acción y las estructuras" (Martuccelli, 2006 , p. 25). Esta recursividad hace referencia a las capacidades que poseen las personas para afrontar las situaciones del contexto y las oportunidades que tienen para fortalecerlas. Es decir, los agenciamientos son prácticas localizadas en la actividad social, en contextos específicos, las cuales generan procesos constantes de producción y reproducción social mediante la "movilización, uso, significación, distribución, transformación, conexión, y también afectación" (Law, 2009, p. 159).

Específicamente, los agenciamientos socio-jurídicos, tienen que ver con la capacidad 
de las personas para actuar con la libertad de hacer y transformar condiciones sociales y/o jurídicas de su contexto, procurándose bienestar, en función de sus metas. Al respecto, Nussbaum (2013) afirma que:

La libertad de agencia de una persona hace referencia a lo que esa persona es libre de hacer y alcanzar en pos de cualquier objetivo o valores que considere importantes. [...] La libertad de agencia es la libertad para alcanzar aquello que la persona -en cuanto agente responsable-decida que debe alcanzar (p. 230).

En conclusión, la capacidad de agencia es un proceso constituyente del sujeto, que se opone a las condiciones que lo determinan y potencia el desarrollo humano.

\section{Materiales y métodos para abordar los agenciamientos socio-jurídicos en mujeres con jefatura de hogar, en contextos de pobreza y vulnerabilidad social.}

Partiendo de la exploración de aspectos tan subjetivos como los proyectos de vida, las experiencias cotidianas, las expectativas, etc., y por otro lado, la necesidad de dar cuenta de las prácticas objetivas de interacción social que un grupo de mujeres con jefatura de hogar y en condiciones de pobreza y vulnerabilidad social, despliegan como estrategias de afrontamiento de las condiciones adversas en las que discurren sus vidas, la selección de un método sensible que posibilite el conocimiento de estas realidades no es una decisión meramente procedimental. Se hace necesario entonces que la investigación socio-jurídica, a partir del giro lingüístico en ciencias sociales, proponga abordajes sensibles de interpretación de las realidades discursivas y reconozca en ellas, su potencial como portadoras de sentido y verdad (Ibáñez, 2003, Nussbaum, 2013), permitiendo develar las formas jurídico-sociales que las sustentan.

Con base en lo anterior, se buscó superar la prevalente visión positivista de validez y cer- teza, sustentada en la cuantificación, en la mera utilización de datos estadísticos o las referencias dogmáticas de las fuentes normativas y/o jurisprudenciales, cuya pretensión resulta limitada para dar cuenta de fenómenos sociales que se ubican en el orden simbólico de las praxis sociales. En consecuencia, la investigación se centró en la comprensión de las narrativas presentes en un grupo de mujeres en jefatura de hogar, las cuales han sido construidas frente a las realidades sociales en las que se desenvuelven, asumiendo que dichas narrativas representan aspectos como las valoraciones morales y políticas que ellas le atribuyen a sus realidades y por tanto, permiten develar los agenciamientos que ellas despliegan. Dichas narrativas fueron exploradas mediante entrevistas semi-estructuradas, observación participante y talleres narrativos, cuyos núcleos de indagación giraron en torno a los contextos de vida en los que se desenvuelven, las percepciones que tienen sobre su comunidad, sobre la intervención del Estado, sobre la eficacia de los programas sociales que reciben y las acciones sociales y jurídicas que ellas despliegan en diferentes órdenes, como estrategias de agenciamiento para solucionar los problemas que le demanda su entorno. El corpus narrativo fue analizado mediante la técnica del análisis cualitativo de contenido, complementado con Atlas-ti, utilizando procedimientos sistemáticos de descripción del contenido discursivo (Bardin, 1996), para así, inferir los conocimientos y las condiciones de producción relativas al sentido de estos textos sociales.

En cuanto a la conformación de la unidad de trabajo, se focalizó un grupo de 34 mujeres con jefatura de hogar, pertenecientes a estratos socio-económicos bajos, vinculadas a diferentes programas sociales como el de madres comunitarias, en el cual trabajan como cuidadoras en hogares infantiles del Instituto Colombiano de Bienestar Familiar (ICBF); el programa "Guardianas de la ladera", un programa de la Alcaldía de Manizales, donde las madres desempeñan el trabajo de cuidadoras del medio ambiente y el grupo de mujeres vinculadas al 
centro de desarrollo comunitario (CEDECO) del barrio Galán, a través del programa "Gota de leche", donde se atienden en comedores comunitarios a personas de la tercera edad. El trabajo de campo se llevó a cabo teniendo en cuenta las consideraciones éticas de protección, anonimato y la no afectación de las mujeres informantes, para lo cual se realizó con antelación una sesión de encuadre con cada grupo y explicación del objetivo del proyecto, además de diligenciar los consentimientos informados con cada una de las mujeres que aceptó participar. Se realizaron 34 entrevistas a mujeres cabeza de hogar, cuyo promedio de edad estuvo en 47 años. Las características básicas del grupo se encuentran representadas en las figuras 1 al 8:

\section{Figura 1. Rangos de edad}

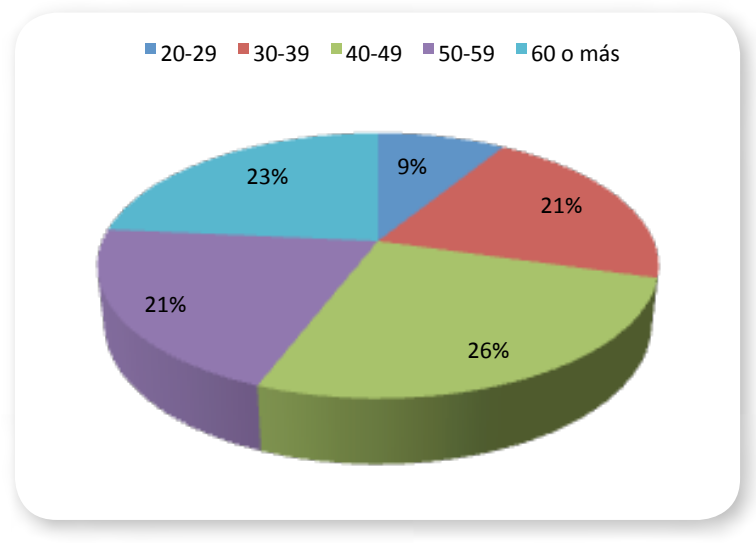

Fuente: elaboración propia.

Figura 2. Nivel de escolaridad

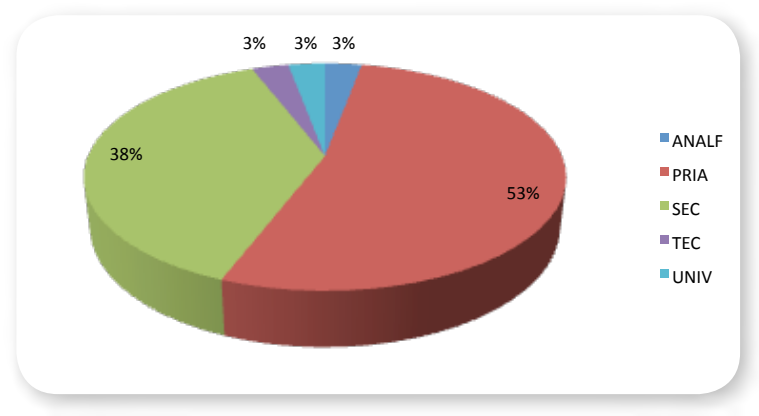

Fuente: elaboración propia.
Figura 3. Desempeña algún trabajo

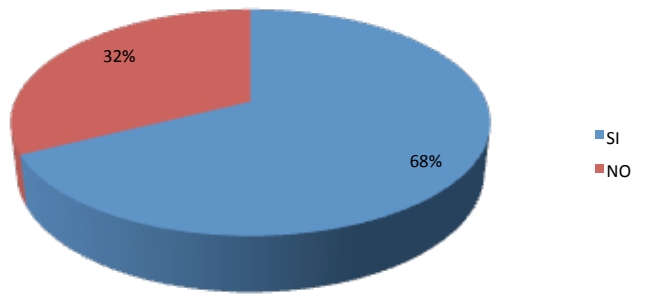

Fuente: elaboración propia.

Figura 4. Tipo de ocupación

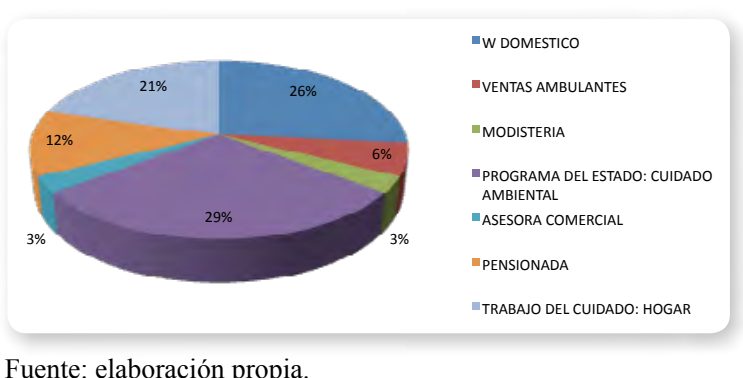

Figura 5. Promedio de ingresos de la mujer cabeza de hogar

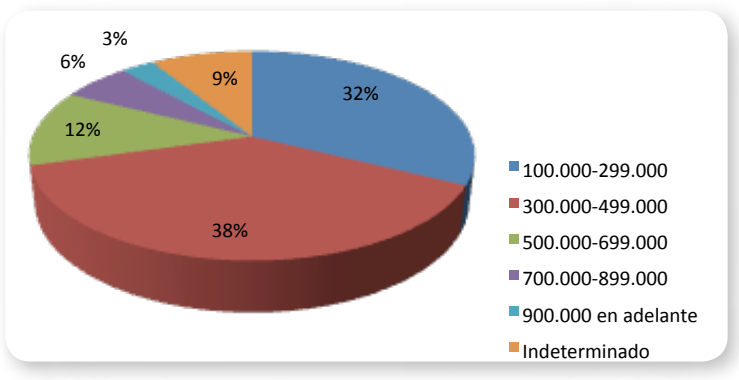

Fuente: elaboración propia.

Figura 6. Estrato socioeconómico

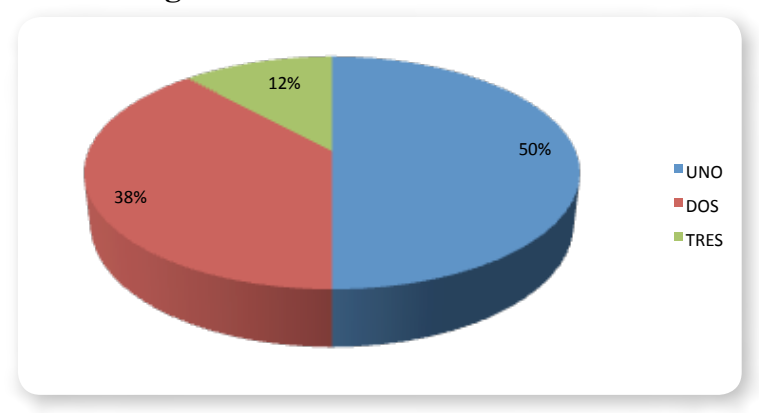

Fuente: elaboración propia. 
Figura 7. Número de integrantes en el hogar

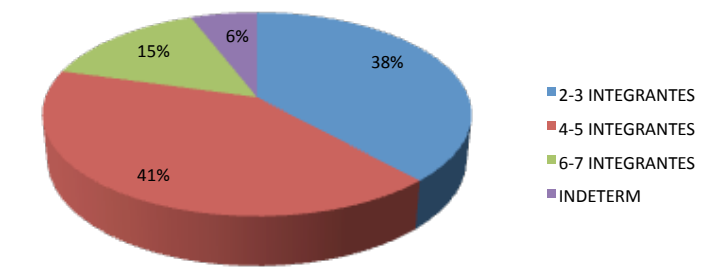

Fuente: elaboración propia.

Figura 8. Vivienda

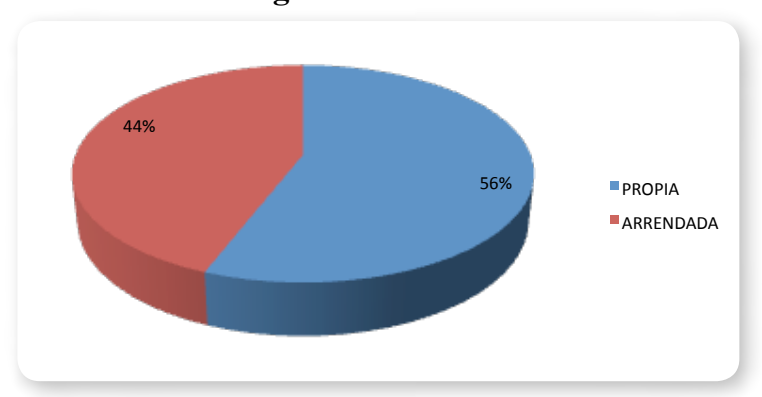

Fuente: elaboración propia.

\section{Hallazgos y resultados}

Los hallazgos obtenidos se refieren específicamente al eje analítico relacionado con las acciones sociales y jurídicas que las mujeres despliegan como estrategias de afrontamiento para solucionar los problemas que le demanda su entorno. Se evidenció una primera práctica de agenciamiento que denominamos agenciamiento social, relacionada con la cultura del cuidado, es decir, ellas movilizan sus recursos personales y actúan buscando transformar sus condiciones objetivas, para lograr un mejor bienestar a partir del sentido preponderante que le otorgan a su función de cuidadoras y protectoras de los otros, incluso, en detrimento de su propio bienestar material.

Soy madre cabeza de hogar, pero no puedo ir a ningún programa porque me queda difícil porque siempre debo cuidar a mi madre y mi hijo. El barrio es sano pero hay lugares cercanos no muy sanos y debo estar pendiente también del niño, sino quien me lo va a cuidar (Comunicación personal, MJH5, E-F7).
Esta práctica de agenciamiento asociada a la cultura del cuidado, se relaciona con las limitaciones de desarrollo humano que tienen estas mujeres, pues el bajo nivel educativo (primaria incompleta) y la poca formación laboral, limita sus oportunidades y las sitúa en desventaja social. Por esta razón, en su mayoría desempeñan actividades laborales relacionadas con la economía del cuidado ${ }^{2}$, referido a "las actividades y prácticas necesarias para la supervivencia cotidiana de las personas en la sociedad en que viven" (Rodríguez, 2015, p. 36), creándose un círculo vicioso donde se profundizan las condiciones de pobreza y exclusión social.

Yo trabajo en oficios varios para dedicarles tiempo a mis hijos; me gusta mucho compartir con ellos. También vendo revistas de Yanbal, Avon, Esika, Carmel, y boletas; me considero buena vendedora, me gusta vender. Hay que rebuscarse la plata, porque está la situación re dura (Comunicación personal, MJH3, E-F3).

Algunas de estas mujeres se ven sometidas a desempeñar actividades laborales informales propias de la llamada "economía del rebusque"3, principalmente asociadas al trabajo del cuidado o a las ventas ambulantes; estas actividades les permite contar con ingresos mínimos para satisfacer las necesidades de subsistencia de ellas y sus familias, y adicionalmente, dadas las características informales de dichas actividades, les posibilita contar con tiempos adicionales para el cuidado del hogar, de sus hijos y en muchos casos, de sus padres ancianos.

Yo no estoy vinculada a ningún programa, mi mamá es la que asiste al CEDECO al programa de la tercera edad de la Gota de Leche, pero yo estoy muy contenta que me la cuiden y ya casi no se enferma. Yo me tengo que quedar arreglando la casa y cuidando a mis hijos y luego vengo y la recojo y bajamos hasta mi ranchito, allá abajo. Esa es mi felicidad, verlos bien a ellos (Comunicación personal, MJH4, E-F10). 
Así pues, se evidenció que el rol social de cuidadoras, se traslapó hacia su actividad laboral, pues todas ellas desempeñan trabajos del cuidado en los hogares infantiles, en el cuidado del medio ambiente o como empleadas domésticas en otros hogares, donde generalmente no reciben una retribución salarial justa, ni gozan de un sistema de protección social. A pesar de ello, estas mujeres ven en este tipo de actividades una ganancia pues logran conciliar los horarios laborales con el tiempo que dedican a su rol de cuidadoras del hogar, ya que ambas cargas están bajo su responsabilidad, produciendo la llamada "doble jornada" con las consecuencias de sobrecarga y limitaciones para su autorrealización. Sin embargo, esta situación no es percibida como una desventaja. Por el contrario, ellas la naturalizan como un deber incuestionable y su rol de cuidadoras lo legitiman en sus aspiraciones relacionadas con el bienestar de los otros, principalmente sus hijos, quienes han quedado bajo su total cuidado. En estas condiciones vitales de existencia, el valor primordial en sus vidas es la ética del cuidado, entendida como la responsabilidad por la vida y protección de los otros, atendiendo a sus necesidades y dirigiendo la acción social a la satisfacción de las mismas.

La ética del cuidado (Gilligan, 1982), basada en los vínculos del afecto y la filiación, pone su acento en la satisfacción de las necesidades del otro y está simbolizada y reproducida culturalmente en el rol de la maternidad. Así lo plantea Castillo (2016):

La mujer-madre todo cuidado y protección. Definición que vuelve transparentes a las mujeres, idénticas así mismas, proyectando en el espacio de la política una definición a priori que las define una y otra vez aferradas a un cuerpo reproductivo. Condición para las políticas de la afirmación de las mujeres que oscurece, sin embargo, historias, cuerpos, sexualidades, políticas y, por paradójico que sea, también identidades (p. 114).
En dicho rol, la función social de la mujer se orienta a la protección y la reproducción. Tal como lo plantea Boff (2012), el cuidado al ser un gesto protector y amoroso hacia el otro y los otros que lo rodean, se convierte en práctica de convivencia pacífica pues "sin cuidado nada de lo que está vivo sobrevive" (p. 15). Esta ética del cuidado revela la capacidad de vivir con y hacia otros, de mostrar preocupación por otros seres humanos y no humanos y de comprometerse con "la mediación, mantenimiento y conservación de la vida" (Carossio, 2014).

Sin embargo, esta ética implica una tensión dialéctica entre los fines de desarrollo de las mujeres y los de la sociedad, pues esta responsabilidad afectuosa y solidaria se contrapone en gran medida a la ética capitalista cimentada en una visión utilitarista de maximización de beneficios y de intercambios exactos. Esta ética del cuidado, al no hacer parte de los valores predominantes de la sociedad capitalista, es relegada al ámbito privado, a las actividades del hogar desempeñadas por las mujeres, donde queda invisibilizada y desvalorizada como producción social.

En la ética del cuidado, el valor de la solidaridad se convierte en un mecanismo para equilibrar las carencias materiales en las cuales viven dichas mujeres. En la mayoría de las veces, se activa a partir de los propios recursos, pero en otros casos se dirige a la posibilidad de ayudar a personas de su comunidad a través del acceso a programas de asistencia social del Estado.

$\mathrm{Si}$ puedo yo ayudo a que mis vecinos conozcan programas que pueden ayudarnos; por ejemplo la labor con los viejitos que realiza la Gota de Leche en el CEDECO del barrio Galán, como lo hace mi mamá que es beneficiaria (Comunicación personal, MJH4, E-, F28).

Cuando alguien necesita un favor, yo lo ayudo sobre todo en temas de vueltas de hospital o de medicamentos, o que necesite un alimento, nada más (Comunicación personal, $\mathrm{MJH} 2$, E-, F14). 
En el caso de la gestión de beneficios de programas estatales, se observó que solo algunas mujeres reconocidas como lideresas en la comunidad despliegan su capacidad de agencia. Ellas movilizan sus propias fuerzas productivas pero también gestionan la oferta de programas sociales del Estado, tales como restaurantes escolares, comedores para la tercera edad, "Familias en acción"4, etc., mostrando tener una mayor capacidad de interlocución pública. En estos casos, la cultura del cuidado adquiere una dimensión política, impregnando las relaciones sociales de una ética de la responsabilidad y cuidado, lo cual convierte al "cuidado en un tema politicamente relevante" (énfasis del autor, Carossio, 2014).

Las condiciones que potencian los agenciamientos de estas mujeres tienen que ver con su autoestima; la imagen de sí mismas como sujetos autónomos que pueden movilizar recursos para mejorar la calidad de vida, las fortalece y les da confianza en un futuro distinto y en su posibilidad de intervenirlo. Aunque en dichas mujeres, está muy arraigada la cultura del cuidado como práctica esencial de sus vidas, también surgen aunque débilmente, los anhelos propios de bienestar, donde forjan horizontes para sí mismas y su comunidad:

Al menos el programa me enseñó a crecer como persona, y a valorarme; a nosotras en Bienestar Familiar, nos dan muchos talleres, y nos enseñan a querernos a nosotras, porque si nosotras no nos queremos nadie nos va a querer, y si yo estoy bien, pues voy a estar bien para los demás (comunicación personal, MJH23, E-F18).

En general, estas lideresas despliegan agenciamientos incursionando en lo público mediante el trabajo comunitario, a través del cual lideran procesos organizativos con mujeres. En estos casos, se constata que han sido potenciados mediante capacitaciones en materia de defensa de sus derechos, brindadas por instituciones estatales. Aunque algunas han usado los mecanismos de defensa de los derechos tales como la acción de tutela, principalmente para lograr el reconocimiento de derechos como la salud o la cuota alimentaria (en los casos en que el padre no cumple), en general, acuden muy poco a estos mecanismos y no parecen empoderarse suficientemente de estos conocimientos.

Yo nunca he hecho nada legal; no he tenido la necesidad, pero mi tía sí, con un primito, por lo de la pierna que no lo querían atender, necesitaba urgente una platina y ella hizo un tutela y yo le ayudé a hacer las vueltas y sí le sirvió (comunicación personal, MJH2, E-F13)

Ahora bien, se identificó una segunda práctica que ubicamos como agenciamientos jurídicos, a través de las cuales algunas mujeres, con conocimientos básicos sobre los mecanismos de defensa de derechos y con la ayuda de profesionales con conocimientos legales, han reclamado mediante la tutela o el derecho de petición, la intervención estatal en casos de abusos por parte de empresas de servicios públicos, la protección del derecho a la salud o la inasistencia alimentaria a sus hijos por parte de los padres. Al respecto, el siguiente relato:

He utilizado la tutela en el caso de la vivienda donde estamos; en la casa de justicia nos han ayudado. También tengo otros dos casos de vecinas que les estoy ayudando; a ellas les ha tocado demandar al cónyuge, para que les ayuden con la alimentación de los hijos (comunicación personal, MJH4, E-F8).

Estos agenciamientos jurídicos se limitan a la esfera familiar y no trascienden a la comunidad. Incluso muchas de estas mujeres renuncian a utilizar dichos mecanismos por considerarlos ineficaces, por ejemplo, en el caso de la inasistencia alimentaria por parte de los padres. Esto es particularmente relevante, pues al parecer, este desempoderamiento jurídico, conlleva una fuerte crítica y desconfianza de las mujeres a la eficacia del orden jurídico establecido; señalando una contra-reacción, una forma de resistencia que las mujeres utilizan para oponerse a la gubernamentalidad estatal y la 
imposición de sus formas de individualización; y aunque puede verse como la desactivación de sus fuerzas de intervención sobre sus contextos, en realidad representa una acción de resistencia, en tanto puede convertirse, como lo afirmó Foucault "en un instrumento para quienes luchan, resisten y no quieren más lo que es" (2012, p. 18).

En este sentido, consideramos que el desempoderamiento jurídico, conlleva una negativa de las mujeres a utilizar, en ciertas circunstancias, los instrumentos jurídicos formales, y debe renombrarse como "resistencia al régimen de gubernamentalidad", pues en suma, se trataría de un tipo de agenciamiento que se convierte, como ya lo dijo Ortner (citado en Mora, 2008), una posición contrahegemónica, que se opone a la utilización de mecanismos jurídicos cuyo privilegio implícito, así como el aparato que administra justicia, están dispuestos para no viabilizar eficazmente las demandas y derechos de estas mujeres. Por esta razón, hay casos en que ellas, a pesar de conocer los instrumentos con los que cuentan para defender sus derechos, no los utilizan y prefieren apelar a prácticas informales de conciliación, generando alternativas al orden establecido. En cualquier caso, la agencia estaría moldeando las subjetividades, en tanto que determina las formas como están siendo en el mundo.

Bajo esta óptica, el desempoderamiento jurídico, que en realidad se configura como "resistencia al régimen de gubernamentalidad", puede expresar la oposición de estas mujeres, a la gubernamentalidad ejercida, tal como lo afirmó Foucault (2009), como una tecnología de poder que se traduce en una suerte de mecanismos de regulación de sus vidas, a través de formas que implican el derecho de hacer vivir de una determinada manera. Este derecho, ejercido sobre ellas, nuevamente se relaciona con la cultura del cuidado, la cual engloba el cuidado material que implica un trabajo; el cuidado económico, que implica un costo y el cuidado psicológico que implica un vínculo afectivo.
El cuidado se convierte en un régimen biopolítico, legitimado por la gubernamentalidad estatal y con una preponderancia en sus vidas, cuya implicación es la responsabilidad total del cuidado de sus hijos asumiendo el rol de cuidadoras y proveedoras, liberando de paso, la responsabilidad del padre-Estado. Por ello, la "resistencia al régimen de gubernamentalidad", también significa la oposición a las relaciones patriarcales de subordinación y dominación en las que han sido construidas. "No he tenido otros casos legales, incluso nunca demandé por alimentos al papá de mis hijos porque ¿para qué? Yo puedo sola" (comunicación personal, MJH15, E-F9).

Lo anterior solo es posible comprenderlo, tomando en cuenta la preponderancia del sistema patriarcal y la cultura del cuidado como tecnología de poder, amparada en el gobierno desde el cual se forman las visiones y aspiraciones de estas mujeres. Al respecto, Nussbaum advierte sobre la necesidad de comprender situadamente, la posición que ocupan ellas en la sociedad, pues "sigue siendo crucial comprender cómo el contexto marca tanto la elección como la aspiración" (2012, p. 64). Esto nos lleva a plantear que respecto a los agenciamientos que las mujeres de este estudio ponen en marcha, tanto la elección como la aspiración que los dinamiza, tienen que ver con su posición dentro de un sistema patriarcal y capitalista que las oprime.

De otra parte, en las prácticas sociales de dichas mujeres, relacionadas con el afrontamiento de las condiciones de riesgo e inseguridad urbana que perciben en sus contextos, despliegan agenciamientos socio-jurídicos. Esto se relaciona con lo planteado por Foucault sobre las «sociedades de seguridad», que operan bajo la gubernamentalidad, donde la forma prevalente de regulación social, es el gobierno del Estado, la cual "es a la vez interior y exterior al Estado, puesto que las tácticas de gobierno son las que permiten definir en cada momento lo que le debe y lo que no le debe concernir; lo que es público y lo que es privado, lo que es estatal y lo que no lo es" (2009, p. 12). 
Desde esta gubernamentalización se establecen las topografías sociales donde los sujetos se relacionan, se regulan y resuelven sus litigios. Bajo este orden, estas mujeres reconocen la validez del Estado como garante del orden y la seguridad y están dispuestas a acudir a instancias de control estatal para afrontar los riesgos que atentan contra la seguridad de su familia. Aunque este tipo de agenciamientos hayan sido puestos en marcha en muchos casos no han encontrado respuesta efectiva en el Estado, lo cual ha generado desconfianza. El siguiente relato da cuenta de estos efectos:

Hace poquito me chuzaron uno de los muchachos; casi me lo matan y fui y coloqué el denuncio en la SIJIN y fui y eso fue como echarlo al agua porque eso no hicieron nada. $\mathrm{Me}$ gané problemas para nada, entonces aprende uno a manejar las cosas de otra forma (comunicación personal, MJH12, E-F20).

Contrario a la situación anterior, los hallazgos mostraron otro marcador que tiene que ver con la potenciación de los agenciamientos socio-jurídicos en función de condiciones subjetivas presentes en dichas mujeres. La principal de estas condiciones se refiere a la capacidad de "revelarse en público", es decir, aparecer como ciudadanas en la esfera pública (Arendt, 1995), liderando procesos motivados por la solidaridad colectiva, a la cual le atribuyen una efectividad mayor que la imputada a los mecanismos legales:

Mire, hemos hecho talleres y brigadas en compañía de la policía cívica; ellos les dictan talleres a los jóvenes, a las familias que están desprotegidas, digámoslo así, y otra entidad de salud hace visitas periódicas, a todas las familias y ahí detectan a las familias con falencia y les dan apoyo psicológico, terapéutico; esos programas ayudan pero yo soy la que anima a la comunidad a participar en ellos, porque si no, no se mueven (comunicación personal, MJH30, TN-F24).
En esta clave, los agenciamientos sociojurídicos pueden ser considerados como medio para la circulación de la voz de estas mujeres en el ámbito público, permitiendo su empoderamiento y la integración con otros actores sociales, mejorando la cohesión social, la confianza y autonomía frente a la toma de sus propias decisiones. Estos agenciamientos, revelan una práctica (praxis) y un discurso (lexis), cuyo ejercicio les permite la integración con los demás, adquiriendo una identidad reconocida comunitariamente.

Con el trabajo de madre comunitaria tengo, porque ahí trabaja uno con la comunidad, ahí solucionamos los problemas de la misma gente; nosotras trabajamos y somos reconocidas en la comunidad porque hablamos por ella y trabajamos por ella (Comunicación personal, MJH34, TN-F17).

Unido a los agenciamientos socio-jurídi$\cos$, en las narraciones aparece una categoría relacionada con la autopercepción comunitaria, la cual actúa como soporte del débil tejido social y a su vez, como vehículo para el fortalecimiento de los agenciamientos. Las mujeres de este núcleo, son percibidas como trabajadoras y honestas y esta valoración la hacen extensiva hacia su propia autoimagen que han puesto toda su existencia en función del trabajo para otros, con una mística excepcional que solo puede explicarse en una fuerte ética del deber, reivindicándose como mujeres "echadas pa'lante", pero que a su vez, legitima su condición de explotación:

No he necesitado nada legal. Sé que es un derecho de petición y la tutela pero no he necesitado de eso. No necesité ni siquiera para demandar al papá de mi hijo, porque yo puedo sola (comunicación personal, MJH10, E-F14).

En los hallazgos también se encontró la interdependencia virtuosa entre las prácticas participativas y los agenciamientos, las primeras se ejercen en pequeños grupos, mediante un ejercicio de reconocimiento afectivo. Es en la cotidianidad de su mundo micro-social donde 
se da la interacción social con su comunidad, contribuyendo a la solución de problemas colectivos locales, por lo cual son reconocidas como lideresas, lo cual las empodera. Esta capacidad de agencia, está presente en algunas de estas mujeres, cuya particularidad es que han estado vinculadas a las mediaciones del Estado, a través de procesos de capacitación en diferentes temáticas como derechos humanos, participación comunitaria, etc. Las mediaciones estatales, son complementarias a la acción de programas sociales subvencionados, donde algunas lo perciben como intervenciones asistencialistas por parte del Estado, las cuales no dan relevancia al componente formativo.

No pertenezco a ningún grupo; participo en reuniones donde los funcionarios invitan a la comunidad pero porque mi mamá pertenece al programa "Gota de Leche"; no he pertenecido a esos grupos porque me quitan mucho tiempo para trabajar, entonces las ayudas que se las den a mi mamá (comunicación personal, MJH22, TN-F7).

Finalmente, dentro de las prácticas asociadas a los agenciamientos socio-jurídicos, aparecen las formas como dichas mujeres resuelven los conflictos en su cotidianidad, que no se circunscriben a mecanismos jurídicos formalizados, pues ellas acuden a sus propias potencialidades discursivas, que activan desde sus visiones éticas del mundo para intervenir dichas realidades y solucionarlas, mediante agenciamientos que buscan reducir el conflicto y la confrontación, bajo formas alternativas y pacíficas de resolución de conflictos, generando cambios no formalizados en lo institucional e incidiendo positivamente en sus mundos cotidianos. Estas mujeres, reconocen la contribución del Estado, en lo concerniente al ámbito de las necesidades de subsistencia, porque su relación ha estado mediada por el asistencialismo, pero en cuanto al mundo micro-social de riesgo e inseguridad, desconfían del orden jurídico instituido. Estas formas alternativas que denominamos formas de pluralismo jurídico, son re- guladoras y legitimadoras del comportamiento social comunitario.

Yo con el diálogo. Yo eso sí soy, eso tengo yo, hablo con los niños. Y con las personas que, con las que está el problema, yo hablo con ellas. Hablo con el papá...porque a veces hacer eso, [lo legal], todo lo enreda más y uno soluciona los problemas mejor con lo que sabemos acá, hablando (comunicación personal, MJH28, TN-F9)

Al respecto, Engle, Griffiths y Tamanaha, plantean que las formas plurales de ordenamiento son dependientes pero al mismo tiempo, están separadas y al ser formas de ordenación, participan en el mismo campo social. En las comunidades de las cuales hacen parte estas mujeres, las tradiciones y visiones éticas no son homogéneas y por ello, recurren a diferentes estrategias para mantener la cohesión o el control social, conservando sus diferencias Este pluralismo jurídico, da cuenta de la compleja red de relaciones que se da en las micro-localidades, cuya diversidad cultural sustenta otras formas de convivir que coexisten en los mismos espacios y con las formas instituidas y legitimadas por el ordenamiento jurídico vigente, "cada uno de estos espacios produce un tipo particular de relaciones sociales, políticas y jurídicas, dando lugar, por lo tanto, a distintos "derechos" (2007, p. 51).

Las estrategias de estas mujeres para afrontar la conflictividad social tiene que ver con el diálogo, la negociación, la reconciliación, la promoción de distintas nociones de justicia bajo mecanismos pacifistas que buscan el entendimiento entre las partes, evitando la intervención del ordenamiento jurídico, aunque reconocen que en algunos casos este es necesario. El despliegue de estos agenciamientos asociados a la convivencia, hacen parte de

[...] procesos sociales que implican complejos vínculos de interdependencia entre las personas, que no son estructuras externas o coercitivas que accionan sobre las personas, sino 
una serie de lazos largos y diferenciados, que se desarrollan a través del tiempo, que supera la perspectiva del individuo "clausus" y de la sociedad como una entidad independiente que se impone a los individuos (Elías, 1989, p. 45).

\section{Conclusiones y discusión}

Los resultados obtenidos en esta investigación sobre los agenciamientos socio-jurídicos de mujeres con jefatura de hogar, revelan diferentes motivaciones que los fomentan o restringen. Dichas motivaciones están representadas en tres dimensiones, a saber:

La primera hace referencia al contexto de pobreza y vulnerabilidad social que caracteriza los entornos de las mujeres, los cuales no solo develan una trama de desigualdades e inequidades donde quedan situadas como sujetos más vulnerables, sino que además las marginaliza respecto a los roles que ejercen tanto en el hogar, trabajo y sociedad. La pobreza desmejora la calidad de vida tanto de ellas como la de sus familias, pero además, las rodea de situaciones asociadas a la inseguridad y riesgo, conflictos familiares y comunitarios y falta de acceso de oportunidades en condiciones de igualdad.

Una segunda dimensión se asocia a la intervención del Estado, la cual, a pesar de ofrecer mecanismos de acceso a la justicia para afrontar las diferentes problemáticas socio-familiares de estas mujeres, no goza de legitimidad o confiabilidad como agente capaz de resolverlas, dejando este espacio a cargo de ellas mismas, quienes por el estereotipo de cuidadoras y pacifistas terminan asumiendo simbólicamente el costo de intervenirlas, desplegando su capacidad de agenciamiento pero también, adjudicándose una carga más en sus vidas.

La pobreza y la ineficaz intervención del Estado, representan dos vías respecto a sus agenciamientos. Por un lado, disminuyen las posibilidades para que las mujeres con jefatura de hogar desarrollen su capacidad de agencia y se desplieguen como sujetos políticos capaces de trasformar su entorno y por otro, dichas condiciones generan fuerzas de oposición respecto a sus entornos, desencadenando posiciones de resistencia emancipadora de los órdenes de dominación que limitan sus agenciamientos y capacidades de transformación.

En medio de esta complejidad, se identificaron cuatro tipos de agenciamientos que son promovidos y a su vez, limitados por los contextos en los que viven dichas mujeres. Estos son: el agenciamiento social asociado a la cultura del cuidado, el agenciamiento jurídico asociado a la convivencia; el desempoderamiento jurídico asociado a la resistencia, al régimen de gubernamentalidad (Foucault, 2009) y el agenciamiento socio-jurídico propiamente dicho, asociado a una tecnología de gobierno.

Respecto al agenciamiento social es concluyente la cultura del cuidado que caracteriza el rol de las mujeres de esta investigación, cuyas acciones de trasformación y cambio están determinadas principalmente por el cuidado de sus familias aun cuando esto implica desatenderse a ellas mismas, sometiéndose a condiciones desventajosas. A pesar de lo anterior, perciben este tipo de agenciamiento como compensación moral derivada del rol materno, denegando los aspectos negativos que pueden incidir en su desarrollo humano.

En cuanto a los agenciamientos jurídicos, estos van más allá de la utilización de instrumentos legales como mecanismos de convivencia y regulación. Para estas mujeres, dichos agenciamientos se convierten en "estrategias de conciliación", que utilizan para enfrentar la violencia que predomina en sus contextos. Es evidente que poseen conocimientos básicos sobre las herramientas jurídicas estatales, no obstante, esto no estimula sus prácticas de agenciamiento, por dos razones: la primera, por la desconfianza que tienen en las instituciones del Estado y la segunda, por el temor de represalias de sus exparejas. Estas condiciones dan lugar a diver- 
sas posturas: en algunas mujeres se estimula la resistencia critica al sistema, estimulando prácticas de agenciamiento alternas y en otras, lamentablemente se provoca una suerte de desesperanza que las aísla de la comunidad y las lleva a asumir una participación no comprometida frente a ella.

Por último, las prácticas de agenciamiento socio-jurídico presentes en muchas de estas mujeres, develan su condición de liderazgo pero siempre bajo un sistema de gubernamentalidad que las produce como sujetos políticos, enmarcadas dentro de las prescripciones estatales, donde se demarca un espacio público, en el cual ellas pueden desplegar sus agenciamientos, empoderándose no solo de roles comunitarios sino de autopercepciones y reconocimiento de su propia identidad, la cual no escapa a la diferenciación de roles sociales y jerarquizaciones patriarcales que ellas incorporan mediante configuraciones poderosas, adecuadas al sistema hegemónico.

Aun cuando el orden patriarcal se reconozca como hegemónico, como todo sistema, no es completamente eficaz en sus cierres y deja líneas de fuga en las divisiones establecidas entre lo público/privado, lo familiar/comunitario, el adentro/afuera, etc., de tal suerte que podemos encontrar que dichas mujeres construyen día a día sus vidas, no en una línea secuencial determinada, sino en modos flexibles, irregulares de afrontamiento de sus condiciones de ser y existir, unas veces dentro del espacio gubernamentalizado donde se precariza su existencia y otras, por fuera de dicho espacio, desde la resistencia critica al sistema, donde despliegan agenciamientos que las empodera y las libera del destino inquebrantable de su condición de procreadoras y las lleva a construir horizontes de sentido, emancipados a partir de los cuales reivindican su lugar en la sociedad como sujetos de derechos.

\section{Referencias}

Arendt, H. (1995). La condición humana. México: Paidós.

Ballén, R. (2007). Ilegitimidad del Estado. Reforma radical o revolución de la diversidad (2a. ed.). Colombia: Editorial Carrera Séptima.

Bardin, L. (1996). Análisis de contenido. Madrid: Akal Ediciones.

Bianciardi, M.(2009).Complejidaddel concepto de contexto. Chile: Pontificia Universidad Católica de Chile. Recuperado de: http://eqtasis.cl/wp-content/ uploads/2018/02/Bianciardi-MarcoComplejidad-concepto-de-contexto.pdf

Boff, L. (2002). El cuidado esencial: Ética de lo humano, compasión por la Tierra. Madrid: Editorial Trotta.

Castillo, A. (2016). Feminismos de la (des) identificación poscolonial latinoamericana. En K. A. Bidaseca (Coord.). $1^{\text {a }}$ Ed. Buenos Aires: CLACSO - IDAES.

Carossio, A. (2014). El aporte de la ética feminista del cuidado para una sociedad sin violencia. Recuperado de: http://www.alainet.org/es/active/70481

Dreze, J, y Sen, A. (1989). Hunger and Public Action. Oxford: Oxford University Press.

Elías, N. (1989). El Proceso de la Civilización. México. Fondo de Cultura Económica.

Engle, S. Griffiths, J., y Tamanaha, B. (2007). Pluralismo Jurídico. Colombia: Siglo del hombre Editores.

Foucault, M. (2006). Seguridad, territorio, población. Buenos Aires: Fondo de Cultura Económica.

Foucault, M. (2012). El poder una bestia magnifica: Sobre el poder, la prisión y la vida. Buenos Aires: Siglo XXI. 
Gilligan, C. (1982). In a Different Voice: Psychological Theory and Women's Development. Cambridge: Harvard University Press.

Ibáñez, T. (2003). El giro lingüístico. En L. Íñiguez (Ed.) Análisis del Discurso. Manual para las ciencias sociales. Barcelona: Editorial, Editorial UOC.

Kagitcibasi, C. (2005). Autonomy and relatedness in cultural context. Implications for self and family. Journal of Cross-Cultural Psychology, 36(4), 403-422. doi: $10.1177 / 0022022105275959$

Law, J. (2008). Actor-network theory and material semiotics. En Bryan S. Turner, (Ed.) The New Blackwell Companion to Social Theory, 3a Edición. Oxford: Blackwell, p.p. 141-158.

Lezama, J. L. (2014). Teoría social, espacio y ciudad. México: Colegio de México.

Martínez, M, y Cubides, J. (2012). Sujeto y política: vínculos y modos de subjetivación. Revista Colombiana de Educación, (63), 67-88.

Martuccelli, D. (2006). Lecciones de sociología del individuo. Recuperado de: http://departamento.pucp.edu.pe/ ciencias-sociales/files/2012/06/ Martuccelli-Lecciones_de_sociologia del_individuo2.pdf

Montaño, S. (2003). Políticas para el empoderamiento de las mujeres como estrategia de lucha contra la pobreza. En R. Atria, M. Siles, I. Arriagada, L. Robinson, y S. Whiteford (Comp.). Capital social y reducción de la pobreza en América Latina y el Caribe: en busca de un nuevo paradigma (pp. 361-377). Santiago de Chile: CEPAL.

Mora, A. S. (2008). Cuerpo, género, agencia y subjetividad. V Jornadas de Sociología de la UNLP, 10, 11 y 12 de diciembre de
2008, La Plata, Argentina. En Memoria Académica. Recuperado de:

http://www.memoria.fahce.unlp.edu.ar/ trab_eventos/ev.6266/ev.6266.pdf

Nussbaum, M. (2012). Las mujeres y el desarrollo humano. España: Editorial Herder.

Nussbaum, M. (2012). Crear capacidades. Propuesta para el desarrollo humano. España: Paidós.

Posas, M. (s. f.). Ciudadanía y desarrollo humano. Honduras: Programa de las Naciones Unidas para el Desarrollo PNUD.

Ramírez, P., y Ziccardi, A (2008). Pobreza urbana, desigualdad y exclusión social en la ciudad del siglo XXI. Una introducción. En R. Cordera, P. Ramírez y A. Ziccardi (Coords.). Pobreza, desigualdad $y$ exclusión social en la ciudad del siglo XXI (pp. 23-49).

Rodríguez, C. (2015). Economía feminista y economía del cuidado. Aportes conceptuales para el estudio de la desigualdad. Revista Nueva Sociedad, (256), 30-44.

Sen, A. (1997). El bienestar, la condición de ser agente y la libertad. Conferencias Dewey de 1984. En Autor. Bienestar, justicia y mercado. Barcelona: Paidós.

Sen, A. (2000). Desarrollo y Libertad. Barcelona: Editorial Planeta.

Sztulwark, D., y Duschatzky, S. (2005). ¿Qué puede una escuela? Notas preliminares sobre una investigación en curso. En G. Frigerio y G.Diker (comps.), Educar: ese acto político (pp. 193 - 210). Buenos Aires: Del Estante Editorial.

Torres Castro, C. (2005). Jóvenes y violencia. Revista Iberoamericana de Educación, (37), 55-92.

Uribe, E y González, M. (2007). La protección jurídica de las personas vulnerables. $R e$ vista de Derecho, (27), 205-229. 


\section{Notas}

${ }^{1}$ Se utilizará la siguiente identificación de los fragmentos textuales recogidos en el trabajo de campo: Identificación del sujeto informante, tipo de técnica, ubicación del fragmento relacionado

MJH: Mujer con Jefatura de Hogar (\#)

E: Entrevista ó TN: Taller narrativo

F: Fragmento (\#)

2 Referido a "todas las actividades y prácticas necesarias para la supervivencia cotidiana de las personas en la sociedad en que viven. Incluye el autocuidado, el cuidado directo de otras personas (la actividad interpersonal de cuidado), la provisión de las precondiciones en que se realiza el cuidado (la limpieza de la casa, la compra y preparación de alimentos) y la gestión del cuidado (coordinación de horarios, traslados a centros educativos y a otras instituciones, supervisión del trabajo de cuidadoras remuneradas, entre otros)" (Rodríguez, 2015, p. 36).
3 Término usado en Colombia, para identificar actividades económicas informales, desempeñadas de forma desregulada, que no requieren cualificación laboral por parte de quienes la ejercen y generan ingresos para subsistir.

${ }^{4}$ Programa estatal de subvención alimentaria y otros servicios sociales, dirigido a erradicar la pobreza en población vulnerable como madres cabeza de hogar, población desplazada, etc. 
